\title{
No evidence of neural adaptations following chronic unilateral isometric training of the intrinsic muscles of the hand: a randomized controlled study
}

\section{Introduction}

Strength training (ST) is well known to induce structural alterations in the skeletal muscle which contribute to significant increases in muscle strength and size (Abernethy et al., 1994). Gains in strength following ST are detected not only in those muscles specifically trained but also in the contralateral homologous muscles that were not targeted with exercise, portraying a phenomenon known as "cross-education" (CE) (Munn et al., 2004). Since morphological or enzymatic changes in the untrained muscles were undetected in the initial phases of training (Narici et al. 1989), neural mechanisms have been accounted for the CE effect, with no consensus, however, on a dominant mechanism or site of adaptation (Enoka, 1997; Hortobagyi, 2005; Carroll et al., 2006; Fimland et al., 2009; Kidgell et al., 2011).

Most studies examining the mechanism of $\mathrm{CE}$ effects have been performed on the upper limb due to the ease with which a range of common electrophysiological techniques can be applied, particularly those involving transcranial magnetic stimulation (TMS). In general these studies have shown that following a single session of strength training employing a simple motor task, there is a transient increase in excitability of corticospinal projections in the "trained" hemisphere as well as a smaller increase in the "untrained" hemisphere (Yue and Cole, 1992; Cramer et al 1999; Muellbacher et al. 2000; Perez et al., 2004; Farthing et al., 2005; Carroll et al., 2006; Perez and Cohen, 2008; Camus et al., 2009; Kidgell et al., 2011; Liang et al. 2014; Leung et al., 2015). The latter have been interpreted as one possible mechanism underlying the $\mathrm{CE}$ effect.

However, the data are less clear in experiments testing the effects of longer term training protocols. Some studies report no changes in corticospinal excitabilty in either hemisphere, whereas others find persisting increases in excitability in both hemispheres (Classen et al., 1999; Lee et al., 2009; Hortobagyi et al., 2011; Kidgell et al., 2015). Changes in intracortical excitability as measured with short-interval intracortical inhibition (SICI) and interhemispheric inhibition (IHI) have also been debated both in acute (Warbrooke and Byblow, 2004; Duque et al., 2008; Vercauteren et al., 2008) and chronic studies (Hortobagyi et al., 2011; Kidgell et al., 2015). However, direct comparisons between these studies are difficult because of differences in muscle studied and the training provided. So, the question remains whether chronic training involving a simple resistance motor 
task is capable to cause significant and meaningful contralateral strength gains and whether they are paralleled by noteworthy supraspinal neural adaptations. From a functional (homeostatic?) perspective, we hypothesize that the neurophysiological changes associated to acute training are unlike to persist following chronic exercise, since they would engage motor and learning circuits, which would be otherwise available for new tasks. Therefore, the aim of the present study was to explore a very wide range of sensory-motor pathways after chronic CE to test if long term changes occur following a 4-week maximal-intensity unilateral isometric training of the first dorsal interosseus (FDI) muscle.

\section{Materials and methods}

\section{Study design and participants}

This was a parallel-group case-control study in a randomized 1:1 allocation ratio. Thirty-four healthy individuals volunteered for the study $(\mathrm{M}: \mathrm{F}=23: 11$; mean age 25.5 \pm 6.0 years; weight $67.6 \pm 11.1 \mathrm{~kg}$; height $170.3 \pm 9.4 \mathrm{~cm}$ ). All subjects were right handed according to the Edinburgh Handiness Inventory. Participants were recruited from the university student population and staff. None of the participants had a history of neurological and/or psychiatric diseases and/or upper limb musculoskeletal disorders, neither presented contraindications to undergo transcranial magnetic stimulation (TMS) and/or surface electrical stimulation procedures. After baseline evaluation, 34 envelopes were numbered consecutively and randomly assigned to an intervention (Training; $n=17$ ) or to a no-intervention (Control; $n=17$ ) group, with a blocking procedure employing Research Randomizer 3.0 software. Testing procedures were performed before (PRE) and after (POST) the experimental period by the same operator at the same time of the day. Both outcome assessors and statistician were blinded to the allocation group. All subjects gave written informed consent to the experimental procedure, which was approved by the local ethics committee. The study was performed in accordance with the Declaration of Helsinki. Data were collected and stored at the Department of Biomedical Sciences, University of Sassari (January-July 2015).

\section{Strength assessment}

Participants were seated in front of a computer screen with feet flat on the floor, back straight and supported by a backrest, shoulders adducted and neutrally rotated, elbows at $90^{\circ}$ and forearms in a neutral position supported by the chair armrests. The wrist was positioned between 0 and $30^{\circ}$ of 
dorsiflexion and between 0 and $15^{\circ}$ of ulnar deviation (Pryce, 1980). Prior to the baseline assessment, all participants attended a preparatory session to familiarize with positioning and testing/training procedures. A light warm-up consisting of 2 trials performed at submaximal intensity (5-s duration each) preceded the testing procedures. Subjects were then required to exert three 5-s maximal voluntary isometric contractions (MVIC) for each motor task performed, with 1 minute of inter-trial rest.

To assess the strength of the FDI muscle, the $3^{\text {rd }}, 4^{\text {th }}$ and $5^{\text {th }}$ fingers were extended and restrained in a cast with Velcro straps, as well as the wrist. For the criterion test the subject was asked to hold a force transducer (P200 Biometrics Ltd, Newport, UK) between the pad of the thumb and the lateral aspect of the middle phalanx of index finger (key or lateral pinch) and between the tips of the thumb and index fingers (fingertip precision grip or tip pinch). Due to its insertion onto the tubercle of proximal phalanx, the strongest activity of the FDI is in key pinch; the FDI is also highly active in tip pinch where it mainly acts as a flexor at the metacarpophalangeal joint (Schreuders et al., 2007). To control for the specificity of the training-induced changes, the pinches between the thumb pad and the pads of index and middle fingers (tripod or palmar pinch) were also tested. To be maintained in the horizontal plane, the wrist and the fingers pinching the transducer rested on a Plexiglas support base affixed to the chair's armrest with a Velcro strap. The force transducer was connected to a data acquisition system (DataLOG MWX8, Biometrics Ltd, Newport, UK). After FDI strength testing was completed, a 10-minute rest was allowed for full recovery and then the handgrip strength, was also assessed with an electronic handgrip dynamometer (G200 Biometrics Ltd, Newport, UK) to verify spatial specificity of the contralateral transfer of strenght. The dominant hand was tested first. A 5-minute rest was allowed between the testing of the two sides. For each strength test the scores of 3 successive trials were recorded for each hand. The highest of the 3 strength scores recorded for each test was recorded as the participant's MVIC. The plan of measurements with the timeline of the study is summarized in Fig. 1.

\section{Contralateral transfer of muscle strength}

To control and estimate the familiarization/learning-effect and obtain net changes in muscle strength following the intervention the equation by Carroll et al. (2006) was employed. For each side the mean strength score recorded in the controls was therefore subtracted from the mean score of the cases. 


\section{Neurophysiological assessments}

Subjects sat in a comfortable chair with the neck supported and were asked to keep their eyes open and to stay relaxed but alert during data collection.

Electromyography (EMG). TMS-evoked motor potentials (MEP) at rest and EMG recruitment, at the MVIC, were bilaterally recorded (D360 amplifier; Digitimer Ltd, Welwyn Garden City, UK) from the FDI muscle using 9-mm-diameter $\mathrm{Ag}-\mathrm{AgCl}$ electrodes in a belly-tendon montage. EMG signals were amplified (×1000), filtered (bandpass 3-3000 Hz) and sampled $(6 \mathrm{kHz})$ using a CED 1401 (Cambridge Electronic Design, Cambridge, UK) power analog-to-digital converter. Raw EMG recordings were rectified for off-line measurements.

TMS. Magnetic stimuli were generated by Magstim 200 stimulators connected in a Bistim module (Magstim Co., Whitland, Dyfed, UK) and delivered in separate sessions to the hand contralateral and ipsilateral primary motor cortex (cM1 and iM1, respectively) through a figure-of-eight coil (external loop diameter of $9 \mathrm{~cm}$ ) with the handle pointing backwards and laterally at $45^{\circ}$ away from the midline. The optimal stimulation site for activation of the contralateral FDI was marked on the scalp with a soft tip pen to ensure that the coil remained in the same place throughout the experiments. Motor threshold was expressed as a percentage of the maximum stimulator output (MSO). The test stimulus (TS) intensity was the intensity sufficient to evoke a $1 \mathrm{mV}$ MEP in the relaxed FDI and, in all trials, frequency of TMS was randomized to $0.2 \mathrm{~Hz} \pm 10 \%$ (one pulse every $4.5-5.5 \mathrm{~s})$.

Electrical stimulations $(E S)$. To test sensorimotor integration, the median nerve ipsilateral to the recorded FDI was electrically stimulated at the wrist (Digitimer DS7A constant current stimulator) through single square-wave pulses $(0.2 \mathrm{~ms}, 0.25 \mathrm{~Hz})$ at an intensity nearly $2-3$ times the perceptual threshold (PT), just above the motor threshold for evoking a visible twitch of the thenar muscles.

\section{Neurophysiological protocols}

Neurophysiological protocols were applied to the hand iM1 and cM1, according to the most acknowledged guidelines (Rossini et al., 2015). Resting motor threshold (RMT) was determined as the minimum stimulus intensity required to produce MEPs $>50 \mu \mathrm{V}$ peak-to-peak amplitude in at least 5 out of 10 consecutive trials in the relaxed muscle. Active motor threshold (AMT) was established as the minimum stimulus intensity able to evoke MEPs $>200 \mu \mathrm{V}$ peak-to-peak amplitude in at least 5 out of 10 consecutive trials during isometric contraction of the tested muscle at $10 \%$ of MVIC. Central motor conduction time (CMCT) was calculated subtracting the mean latency of the $1 \mathrm{mV}$ MEP induced by TMS of the cervical spinal root at C7 level from the mean 
latency of the $1 \mathrm{mV}$ MEP elicited cortical TMS. The RC curve was constructed plotting peak-topeak amplitudes of mean MEPs induced by a single-pulse TMS delivered at intensities from 90 to $150 \%$ of RMT. Seven blocks of 10 stimuli each were collected. SICI and intracortical facilitation (ICF) were assessed with a conditioning stimulus (CS) of $80 \%$ RMT preceding a TS of $1 \mathrm{mV}$ MEP by 3 and $10 \mathrm{~ms}$, respectively. Short-interval intracortical facilitation (SICF) was tested pairing stimuli of $1 \mathrm{mV}$ MEP and $80 \%$ RMT intensities at an inter-stimulus interval (ISI) of $1.5 \mathrm{~ms}$. Longinterval intracortical inhibition (LICI) was evaluated pairing stimuli of $1 \mathrm{mV}$ MEP at ISIs of 100 and $200 \mathrm{~ms}$. Short and long latency interhemispheric inhibition (IHI) were tested using $7 \mathrm{~cm}$ double coils (model $\mathrm{D}^{2}{ }^{2}$ ) and delivering a CS of $1 \mathrm{mV}$ MEP to the hand M1 of one side, 10 and $40 \mathrm{~ms}$ before the administration of a TS of $1 \mathrm{mV}$ MEP to the $\mathrm{cM} 1$, respectively. EMG recruitment was also assessed as an index of cortico-spinal excitability by measuring the Root Mean Square (RMS) in the rectified raw EMG recorded during 1 second MVIC of the FDI (5 trials for each muscle).

Cortical sensorimotor integration was bilaterally assessed measuring short- and long-afferent intracortical inhibition (SAI and LAI, respectively). SAI and LAI were tested coupling the ES of the median nerve with TMS of the cM1 at ISIs of 20 and $200 \mathrm{~ms}$, respectively. For each trial of the above protocols 10 MEPs (test MEP or conditioned MEPs at any ISI) were randomly recorded and averaged. Peak-to-peak amplitude was measured for each MEP and that of conditioned MEPs was expressed as a ratio of the averaged test MEP.

\section{Intervention}

A maximal-intensity isometric training of the right dominant FDI was administered to the training group. The participants were seated in the same chair employed for the testing procedure and consistently positioned. The training protocol consisted of a 4-week unilateral training, 3 days/week on non-consecutive days, with a 20-minute duration per session. After a light warm-up (see above) subjects performed 5 sets of 10 isometric key or lateral pinch contractions holding the same force transducer employed for the assessments between the pad of the thumb and the lateral aspect of the middle phalanx of index finger. The duration of the contraction and the inter-contraction rest were both 5-s long. To avoid the onset of fatigue a 3-minute recovery was allowed among sets. During exercise participants were provided with a visual feedback displaying the real-time level of strength reached to motivate the achievement of maximal performance. To minimize any mirror activity in the untrained FDI, subjects were instructed to keep this muscle completely relaxed and were helped in achieving this goal by an auditory feedback of left FDI EMG activity. Volunteers assigned to the 
control group attended the laboratory for the same number of sessions and placed in the same standardized position for the 20-minute session duration without performing any exercise.

\section{Data analysis}

Statistical analysis was performed using the SPSS 18 software (SPSS Inc., Chicago, IL, USA). An $a$ priori power analysis assuming conservatively an expected effect size (Cohen's $d$ ) of 0.5 and a statistical power of 0.80 at a 0.05 alpha level, was performed, resulting in a sample size of 17 subjects per group. Compound symmetry of data was evaluated with the Mauchly's sphericity test. The Greenhouse-Geisser correction was used to compensate for non-spherical data. Demographic and anthropometric variables (age, weight, height, gender) were analyzed at baseline with one-way ANOVA or Chi-Square test, when appropriate. The Shapiro-Wilk test was used to assess normality. Homogeneity of variances between groups at baseline for dynamometric variables was evaluated by Levene's test.

Dynamometric variables - PRE to POST changes in the dynamometric parameters were analyzed with a repeated-measures analysis of variance (ANOVA) with GROUP (training, control), TIME (PRE, POST) and SIDE (dominant, non-dominant) as factors. When significant $F$ values were observed, pairwise comparisons with Bonferroni adjustment were used. Cohen's $d$ effect size magnitudes (small $\leq 0.5$; moderate $0.51-0.79$; large $\geq 0.8$ ) were also used to quantify differences in the data after intervention. Only for dynamometric variables test-retest relative reliability was assessed by 2-way random intra-class correlation coefficient for single measures $\left(\mathrm{ICC}_{2,1}\right)$. The ICC coefficients were calculated taking a value $<0.4$ as an index of poor reliability, 0.4 to 0.75 fair to good reliability, and $>0.75$ excellent reliability (Shrout and Fleiss, 1979). Absolute reliability was estimated with the coefficient of variation $(\mathrm{CV} \%)$ and with the standard error of measurement (SEM) (Weir, 2005). The responsiveness of strength measurements to training was estimated by calculating the individual smallest real difference (SRDi) which serves as a cutoff threshold for meaningful change (Lexell and Downham, 2005).

Neurophysiological variables - Repeated-measures ANOVA was used to assess the effect of training (PRE, POST), SIDE (dominant, non-dominant) and GROUP (training, control) for RMT, AMT, 1mV MEP, CMTC and EMG. The effect of TMS intensity was evaluated for RC (90-150\% of RMT). For paired-pulse TMS protocols, the effect of training (PRE, POST), SIDE (dominant, non-dominant), GROUP (training and control) and ISI (SICI: $3 \mathrm{~ms}$; ICF: $10 \mathrm{~ms}$; SICF: $1.5 \mathrm{~ms}$; 
LICI: 100 and $200 \mathrm{~ms}$; SAI: $20 \mathrm{~ms}$; LAI: $200 \mathrm{~ms}$, IHI: 10 and $40 \mathrm{~ms}$ ) on the amplitude of the conditioned MEP (expressed as conditioned amplitude/unconditioned amplitude) was assessed by a repeated-measures ANOVA. In case of significant differences, paired $t$ tests were performed applying the Bonferroni correction for multiple comparisons.

For all variables a $p$ value $<0.05$ was considered significant. Unless otherwise stated, values are expressed as mean \pm standard deviation (SD).

\section{Results}

Demographic and anthropometric characteristics of the sample are detailed by group in Table 1.

\section{Dynamometric results}

\section{Reproducibility and responsiveness of strength measurements}

Relative reproducibility of the strength measurements as calculated by ICC coefficients proved very high (all coefficients $>0.8$ ). The analysis of the absolute reproducibility revealed a low variability as assessed by CV\% (range: 2.9-6.4\%) and SEM (range: 0.92-1.26 units). The calculation of the individual smallest real difference in absolute values (SRDi) for each motor task allowed the definition of a cutoff to be exceeded in order to consider the training-induced changes as meaningful in both the right trained (key pinch: $+3.6 \mathrm{~N}$; tip pinch: $+4.2 \mathrm{~N}$; tripod pinch: $+4.9 \mathrm{~N}$; handgrip: $+4.3 \mathrm{~N}$ ) and in the left untrained hand (key pinch: $+3.9 \mathrm{~N}$; tip pinch: $+4.9 \mathrm{~N}$; tripod pinch: +4.7 N; handgrip: $+4.1 \mathrm{~N}$ ). All the reproducibility and responsiveness results are detailed by side and by motor task in Table 2 .

\section{Maximal voluntary strength}

There was no difference between groups at baseline in the levels of strength of the dominant right and non-dominant left hands as tested by tip, key and tripod pinches and by the whole handgrip test (Table 3). Gross and net training-induced changes are detailed by type of motor task and by side in Table 3, with the accompanying ANOVA results in Table 4. The mirror EMG activity in the left FDI, expressed as percent of the MVIC activity, was less than $1 \%$ in all the motor tasks performed.

IN summary, the increase in strength was significantly greater in the trained than in the control (untrained) group for the key pinch and tip pinch tasks, but not the tripod or handgrip tasks (significant GROUP*TIME interaction for the first two tasks only). It should be noted that although the effect was greater in the trained group, even the control group improved in strength in the first 
two tasks, perhaps due to familiarity with the task. The additional increment in strength due to training over control was relatively small $(8.5 \%$ and $7.2 \%$ for key pinch and tip pinch in the trained hand; $6.4 \%$ and $4.7 \%$ in the untrained hand) (see Table 3). The increase in strength was not significantly different on the trained and untrained side (i.e. none of the TIME*SIDE interactions were significant).

\section{Neurophysiological results}

Corticospinal and intracortical excitability - Repeated measures ANOVA showed that RMT, $1 \mathrm{mV}$ MEP and CMCT were not significantly different by TIME and by GROUP. A significant main effect of TIME was detected for AMT but no significant effect of GROUP and GROUP*TIME interaction were found (Table 5).

Repeated measures ANOVA for the RC showed a significant effect $(p<0.0001)$ of TMS intensity (90,100,110,120,130, 140 and 150\% of RMT) but no main effects of TIME, GROUP and no interactions among factors (Fig. 2 and Table 5). Repeated measures ANOVA revealed no main effects of TIME and GROUP in the paired-pulse protocols testing SICF, SICI, ICF,LICI,SIHI and LIHI. A significant main effect of ISI (at ISIs of 1.5, 3, 10, 100 and $200 \mathrm{~ms}$, respectively, and 10 and $40 \mathrm{~ms}$ for SIHI and LIHI) was detected (all $p$ values $<0.001$ ) but no significant interactions among factors (Fig. 3 and Table 5).

Sensorimotor integration at the cortical level - Repeated measures ANOVA revealed no main effects of TIME and GROUP in the paired-pulse protocols testing SAI and LAI. A significant effect of ISI (at ISIs of 20 and $200 \mathrm{~ms}$, for SAI and LAI, respectively) was observed (all $p<0.001$ ) but no significant interactions among factors (Fig. 3 and Table 5).

Overall, no main effect of SIDE was observed for any of the neurophysiological variables analysed. Following the intervention, no main effects of TIME $\left(\mathrm{F}_{1,64}=0.090 ; p=0.76\right)$, GROUP $\left(\mathrm{F}_{1,64}=1.238\right.$; $p=0.27)$ or GROUP*TIME interaction $\left(\mathrm{F}_{1,64}=0.053 ; p=0.82\right)$ were detected in RMS of EMG activity during a MVIC.

\section{Discussion}


In the present study a 4-week maximal-intensity unilateral isometric training increased maximal strength in both the trained and untrained FDI muscles with high spatial and task specificity. The increases in strenght, although significant, occurred at trivial effect sizes and were below the cutoffs for meaningful change. Moreover, strength gains were not paralleled by evidence of direct or crossed cortical or cortico-spinal adaptations in response to the chronic exercise.

\section{Spatial specificity}

A chronic training of the dominant hand with a key lateral pinch motor task induced significant improvements in maximal strength not only in the FDI muscle targeted with exercise but also in the homologous contralateral untrained muscle, depicting a CE effect. Strength adaptations in response to training showed a high spatial specificity depending on the biomechanical features of the motor task. In fact, this phenomenon was observed in the two tasks specifically engaging the FDI but not in those involving only marginally or not involving this muscle, according to the well established specificity of the response to unilateral training (Refs. in Farthing et al. 2005; 2009).

\section{Training-induced changes in strength}

Following the intervention, MVIC increased to a similar extent in both the trained and untrained FDI, with the net contralateral strength gains ranging 4.7-6.4\%, in line with CE magnitudes outlined for the upper limb (Munn et al., 2004; Carroll et al., 2006). A greater extent of CE (40-70\%) has been previously reported but several factors such as heterogeneity in the training regimen, upper limb muscle or study design can account for this discrepancy (Farthing et al., 2009; Hortobagyi et al., 2011). When assessing the extent of the CE effect a between-subject design, like that chosen in the present study, is considered mandatory to control for familiarization and to weigh the traininginduced net change (Carroll et al., 2006). In the trained and untrained FDI we estimated a familiarization/learning-effect among the 4 different tasks of $0.1-5.5 \%$ and $1.4-4.8 \%$, respectively, which, although generally considered small (Carroll et al., 2006), represents a quite relevant fraction of the gross strength gains measured in the ipsilateral trained (7.3-14\%) and in the contralateral untrained (7.8-9.5\%) muscles and needs, therefore, to be taken into proper account since testing itself can improve performance due to habituation of subjects to the test procedure (Gleeson and Mercer, 1996; Manca et al., 2015).

The PRE to POST differences in strength exhibited by both the trained and untrained FDI muscles during the key and tip pinches, despite being significant, appeared not relevant. In fact, although strength levels were higher than baseline, they were associated to between-subjects and between- 
sides very small/small effect sizes (Cohen's $d=0.2-0.4$ ), indicating that the average value of strength after training was only 0.2-0.4 standard deviations above the average value at baseline (Cohen, 1988).

The net strength improvements observed in the trained and untrained FDI muscles did not exceed their thresholds for relevant change as estimated by the SRDi. Taken together, all these data suggest that, in a functional perspective, the isometric training is not capable to meaningfully impact on the level of strength, even when conducted at maximal intensity.

\section{Neural adaptations following a long-lasting training}

As reviewed in the Introduction, there is some debate over the presence and nature of changes in excitability of central motor circuits after chronic strength training (Classen et al., 1999; Lee et al., 2009; Hortobagyi et al., 2011; Kidgell et al., 2015). These discrepancies have been partly explained by factors such as the different nature of the intervention, the body territory and the duration of the training provided. In the present study no changes in any of the neurophysiological parameters describing cortico-spinal and intracortical excitability, IHI and cortical sensory-motor integration were observed in both iM1 and cM1, after 4 weeks of maximal-intensity isometric training. While our findings of an unchanged cortico-spinal and intracortical excitability are in agreement with those reported by Hortobagyi et al. (2011) in the same muscle following a training protocol similar to ours, they are in disagreement with the significant reduction in IHI observed in that study. Interestingly, in both studies any mirror activity in the resting hand was intentionally suppressed, which might have contributed to the evidence of unchanged corticospinal excitability (Sohn et al., 2002). Although further research is required to weigh the role played by the voluntary suppression of mirror movements on CE, compelling evidence has been provided on the apparently paradoxical role played by IHI reduction in preventing mirror EMG activity while chronically mediating CE (Hortobagyi et al., 2011). In fact, it is assumed that during the exercise of one hand, IHI's function is to eliminate unwanted mirror activity in the resting contralateral hand to strengthen inter-limb independence (Hoy et al., 2004; Leocani et al., 2000) and facilitate unimanual movements (Vercauteren et al., 2008). In this perspective, a reduction in IHI from the trained to the untrained hemisphere would make the two limbs more independent, potentially inducing a greater tendency to make mirror movements or impair bimanual coordination. In the absence of any mirror activity, Hortobagyi et al. (2011) found a significant reduction in IHI, whereas we could not observe any change. A couple of differences between Hortobagyi's and our study should be considered: one is 
the baseline level of SIHI (75\% versus 35-40\%), the other is the intensity of the exercise (submaximal versus maximal). Hortobagyi et al. (2011) might have found it easier to reduce IHI since they began with more IHI; additionally, while emphasising the need to maintain the nontrained hand relaxed, we used maximal contraction. This could have paradoxically increased IHI (at least during the training) from trained to untrained hemisphere, counteracting any reductions that might have occurred otherwise.

We also detected no significant physiological changes at the level of a wide range of intracortical circuits regulating cortical excitability and sensory-motor integration either in the resting iM1, as it would have been anticipated with CE paradigms (for reviews: Munn et al., 2004; Carroll et al., 2006), or in the directly "trained" cM1, which is a generally expected outcome in strength conditioning literature (Enoka, 1997). These findings are difficult to interpret in light of the available evidence, which reports that isometric training protocols are able to induce changes in intherhemispheric plasticity (Hortobagyi et al. 2011) and that the voluntary isometric tonic contraction of hand muscles is capable of inducing bidirectional changes in plasticity between homologous areas of the iM1 and cM1 (Gentner et al., 2008; Hortobagyi et al. 2011). Furthermore, the afferent feedback from a muscle targeted with exercise has been reported to induce a reorganization of motor cortex outputs (Lucas and Fetz, 2013). The stability of neurophysiological parameters that we found after a long-lasting period of training, might be viewed as the result of homeostatic plasticity phenomena which may have occurred to ensure the maintenance of stable levels of neural activity over time (Müller-Dahlhaus and Ziemann, 2015). This type of metaplasticity operates in both excitatory and inhibitory intracortical circuits (Murakami et al., 2012) and can last for days (Abraham et al., 2001) and even weeks (Buschler and ManahanVaughan, 2012). We hypothesize that the acute effects, revealed by TMS protocols probing easily modulated motor circuits, may be needed to initiate the CE, while the persisting effects may be consolidated in circuits other than these, to leave them available for other functions and/or learning tasks. In this perspective, the intervention of homeostatic plasticity could restore the physiological balance between the two hemispheres perturbed by the training protocol.

Finally, the experimental conditions here chosen, which focused on supraspinal rather than spinal mechanisms do not allow to exclude as possible contributors any adaptations in spinal excitability which have been previously shown to mediate CE (Hortobagyi, 2005; Fimland et al., 2009). Furthermore, remote cortical regions such as supplementary cortical areas, parietal cortices, basal 
ganglia, cerebellum and brainstem cannot be ruled out (Carroll et al., 2006; Koch et al., 2009; Sehm et al., 2010).

\section{Neural adaptation following a chronic simple motor task}

Although simple motor tasks have been portrayed as capable of inducing a consistent CE effect along with direct and crossed neural adaptations (for reviews: Munn et al., 2004; Carroll et al., 2006), it has been proposed that motor skill acquisition, or motor learning, is a prerequisite factor in driving representational plasticity in M1 (Plautz et al., 2000) and, accordingly, the repetitive execution of a simple movement has been found to induce no functional reorganization of cortical maps in the M1 of monkeys (Plautz et al., 2000) nor considerable M1 chronic adaptations in humans, not even when maximally increasing the force required to execute the task (Carroll et al., 2002). In light of these reports, we argue that besides the temporal factor, the low-demanding nature of the isometric finger pinching task employed in the present study might have played a crucial role in the minor extent of cortico-cortical and/or cortico-spinal adaptations and, indirectly, of the CE effect here observed. In this sense, dynamic training may be superior to isometric training (Morrissey et al., 1995) and, compared to the latter, isotonic contractions result in greater neural adaptations (Schmitz and Westwood, 2001; Yahagi et al., 2003; Kidgell et al., 2015). These heterogeneous findings call for fresh research specifically aimed at comparing the effects of different regimens of muscle contractions (i.e., isometric versus isotonic versus isokinetic) at different intensities (i.e., maximal versus submaximal) and with different durations (i.e., acute versus chronic) on the magnitude of contralateral gains in strength and on their neurophysiological correlates.

\section{References}

1. Abernethy PJ, Jürimäe J, Logan PA, Taylor AW, Thayer RE. Acute and chronic response of skeletal muscle to resistance exercise. Sports Med. 1994 Jan;17(1):22-38.

2. Munn J, Herbert RD, Gandevia SC. Contralateral effects of unilateral resistance training: a meta-analysis. J Appl Physiol (1985). 2004 May;96(5):1861-6. 
3. Narici MV, Roi GS, Landoni L, Minetti AE, Cerretelli P. Changes in force, cross-sectional area and neural activation during strength training and detraining of the human quadriceps. Eur $\mathbf{J}$ Appl Physiol Occup Physiol. 1989;59(4):310-9.

4. Enoka RM. Neural adaptations with chronic physical activity. J Biomech. 1997 May;30(5):44755.

5. Hortobágyi T. Cross education and the human central nervous system. IEEE Eng Med Biol Mag. 2005 Jan-Feb;24(1):22-8.

6. Carroll TJ, Herbert RD, Munn J, Lee M, Gandevia SC. Contralateral effects of unilateral strength training: evidence and possible mechanisms. J Appl Physiol (1985). 2006 Nov;101(5):1514-22.

7. Fimland MS, Helgerud J, Solstad GM, Iversen VM, Leivseth G, Hoff J. Neural adaptations underlying cross-education after unilateral strength training. Eur J Appl Physiol. 2009 Dec;107(6):723-30.

8. Kidgell DJ, Stokes MA, Pearce AJ. Strength training of one limb increases corticomotor excitability projecting to the contralateral homologous limb. Motor Control. 2011 Apr;15(2):247-66.

9. Carroll TJ, Riek S, Carson RG. The sites of neural adaptation induced by resistance training in humans. J Physiol. 2002 Oct 15;544(Pt 2):641-52.

10. Yue G, Cole KJ. Strength increases from the motor program: comparison of training with maximal voluntary and imagined muscle contractions. J Neurophysiol. 1992 May;67(5):111423.

11. Farthing JP, Chilibeck PD, Binsted G. Cross-education of arm muscular strength is unidirectional in right-handed individuals. Med Sci Sports Exerc. 2005 Sep;37(9):1594-600.

12. Liang N, Funase K, Takahashi M, Matsukawa K, Kasai T. Unilateral imagined movement increases interhemispheric inhibition from the contralateral to ipsilateral motor cortex. Exp Brain Res. 2014 Jun;232(6):1823-32.

13. Cramer SC, Finklestein SP, Schaechter JD, Bush G, Rosen BR. Activation of distinct motor cortex regions during ipsilateral and contralateral finger movements. J Neurophysiol. 1999 Jan;81(1):383-7.

14. Muellbacher W, Facchini S, Boroojerdi B, Hallett M. Changes in motor cortex excitability during ipsilateral hand muscle activation in humans. Clin Neurophysiol. 2000 Feb;111(2):3449. 
15. Perez MA, Lungholt BK, Nyborg K, Nielsen JB. Motor skill training induces changes in the excitability of the leg cortical area in healthy humans. Exp Brain Res. 2004 Nov;159(2):197205.

16. Perez MA, Cohen LG. Mechanisms underlying functional changes in the primary motor cortex ipsilateral to an active hand. J Neurosci. 2008 May 28;28(22):5631-40.

17. Leung M, Rantalainen T, Teo WP, Kidgell D. Motor cortex excitability is not differentially modulated following skill and strength training. Neuroscience. 2015 Oct 1;305:99-108.

18. Pryce JC. The wrist position between neutral and ulnar deviation that facilitates the maximum power grip strength. J Biomech. 1980;13(6):505-11.

19. Schreuders TAR, Brandsma JW, Stam HJ The intrinsic muscles of the hand. Function, assessment and principles for therapeutic intervention. Phys Med Rehab Kuror 2007; 17: 20-27.

20. Rossini PM, Burke D, Chen R, Cohen LG, Daskalakis Z, Di Iorio R, et al. Non-invasive electrical and magnetic stimulation of the brain, spinal cord, roots and peripheral nerves: Basic principles and procedures for routine clinical and research application. An updated report from an I.F.C.N. Committee. Clin Neurophysiol. 2015 Jun;126(6):1071-107.

21. Shrout PE, Fleiss JL. Intraclass correlations: uses in assessing rater reliability. Psychol Bull. 1979 Mar;86(2):420-8.

22. Weir JP. Quantifying test-retest reliability using the intraclass correlation coefficient and the SEM. J Strength Cond Res. 2005;19(1):231-240.

23. Lexell JE, Downham DY. How to assess the reliability of measurements in rehabilitation. Am $\mathbf{J}$ Phys Med Rehabil. 2005;84(9):719-723.

24. Farthing JP. Cross-education of strength depends on limb dominance: implications for theory and application. Exerc Sport Sci Rev. 2009 Oct;37(4):179-87.

25. Hortobágyi T, Richardson SP, Lomarev M, Shamim E, Meunier S, Russman H, Dang N, Hallett M. Interhemispheric plasticity in humans. Med Sci Sports Exerc. 2011 Jul;43(7):118899.

26. Gleeson NP, Mercer TH. The utility of isokinetic dynamometry in the assessment of human muscle function. Sports Med. 1996 Jan;21(1):18-34.

27. Manca A, Pisanu F, Ortu E, De Natale ER, Ginatempo F, Dragone D, Tolu E, Deriu F. A comprehensive assessment of the cross-training effect in ankle dorsiflexors of healthy subjects: A randomized controlled study. Gait Posture. 2015 Jun;42(1):1-6.

28. Cohen, J. Statistical power analysis for the behavioral sciences (2nd ed.). Hillsdale, NJ: Lawrence Earlbaum Associates; 1988. 
29. Warbrooke SA, Byblow WD. Modulation of interhemispheric inhibition during passive movement of the upper limb reflects changes in motor cortical excitability. Exp Brain Res. 2004 May;156(1):11-9.

30. Duque J, Mazzocchio R, Stefan K, Hummel F, Olivier E, Cohen LG. Memory formation in the motor cortex ipsilateral to a training hand. Cereb Cortex. 2008 Jun;18(6):1395-406.

31. Vercauteren K, Pleysier T, Van Belle L, Swinnen SP, Wenderoth N. Unimanual muscle activation increases interhemispheric inhibition from the active to the resting hemisphere. Neurosci Lett. 2008 Nov 21;445(3):209-13.

32. Lee M, Gandevia SC, Carroll TJ. Unilateral strength training increases voluntary activation of the opposite untrained limb. Clin Neurophysiol. 2009 Apr;120(4):802-8.

33. Classen J, Liepert J, Hallett M, Cohen L. Plasticity of movement representation in the human motor cortex. Electroencephalogr Clin Neurophysiol Suppl. 1999;51:162-73.

34. Kidgell DJ, Frazer AK, Daly RM, Rantalainen T, Ruotsalainen I, Ahtiainen J, Avela J, Howatson G. Increased cross-education of muscle strength and reduced corticospinal inhibition following eccentric strength training. Neuroscience. 2015 Aug 6(300):566-75.

35. Sohn YH, Jung HY, Kaelin-Lang A, Hallett M. Excitability of the ipsilateral motor cortex during phasic voluntary hand movement. Exp Brain Res. 2003 Jan;148(2):176-85.

36. Hoy KE, Fitzgerald PB, Bradshaw JL, Armatas CA, Georgiou-Karistianis N. Investigating the cortical origins of motor overflow. Brain Res Brain Res Rev. 2004 Nov;46(3):315-27.

37. Leocani L, Cohen LG, Wassermann EM, Ikoma K, Hallett M. Human corticospinal excitability evaluated with transcranial magnetic stimulation during different reaction time paradigms. Brain. 2000 Jun;123 (Pt 6):1161-73.

38. Gentner R, Wankerl K, Reinsberger C, Zeller D, Classen J. Depression of human corticospinal excitability induced by magnetic theta-burst stimulation: evidence of rapid polarity-reversing metaplasticity. Cereb Cortex. 2008 Sep;18(9):2046-53.

39. Lucas TH, Fetz EE. Myo-cortical crossed feedback reorganizes primate motor cortex output. J Neurosci. 2013 Mar 20;33(12):5261-74.

40. Müller-Dahlhaus F, Ziemann U. Metaplasticity in human cortex. Neuroscientist. 2015 Apr;21(2):185-202.

41. Murakami 2012 Murakami T, Müller-Dahlhaus F, Lu MK, Ziemann U. Homeostatic metaplasticity of corticospinal excitatory and intracortical inhibitory neural circuits in human motor cortex. J Physiol. 2012 Nov 15;590(22):5765-81. 
42. Abraham WC, Mason-Parker SE, Bear MF, Webb S, Tate WP. Heterosynaptic metaplasticity in the hippocampus in vivo: a BCM-like modifiable threshold for LTP. Proc Natl Acad Sci U S A. 2001 Sep 11;98(19):10924-9.

43. Buschler A, Manahan-Vaughan D. Brief environmental enrichment elicits metaplasticity of hippocampal synaptic potentiation in vivo. Front Behav Neurosci. 2012 Dec 14;6:85.

44. Koch G, Ruge D, Cheeran B, Fernandez Del Olmo M, Pecchioli C, Marconi B, Versace V, Lo Gerfo E, Torriero S, Oliveri M, Caltagirone C, Rothwell JC. TMS activation of interhemispheric pathways between the posterior parietal cortex and the contralateral motor cortex. J Physiol. 2009 Sep 1;587(Pt 17):4281-92.

45. Sehm B, Perez MA, Xu B, Hidler J, Cohen LG. Functional neuroanatomy of mirroring during a unimanual force generation task. Cereb Cortex. 2010 Jan;20(1):34-45.

46. Plautz EJ, Milliken GW, Nudo RJ. Effects of repetitive motor training on movement representations in adult squirrel monkeys: role of use versus learning. Neurobiol Learn Mem. $2000 \mathrm{Jul} ; 74(1): 27-55$.

47. Morrissey MC, Harman EA, Johnson MJ. Resistance training modes: specificity and effectiveness. Med Sci Sports Exerc. 1995 May;27(5):648-60.

48. Schmitz RJ, Westwood KC. Knee Extensor Electromyographic Activity-to-Work Ratio is Greater With Isotonic Than Isokinetic Contractions. J Athl Train. 2001 Dec;36(4):384-387.

49. Yahagi S, Ni Z, Takahashi M, Takeda Y, Tsuji T, Kasai T. Excitability changes of motor evoked potentials dependent on muscle properties and contraction modes. Motor Control. 2003 Oct;7(4):328-45. 